\title{
MÉTAPHORES ET CALQUES DANS LA CRÉATION PHRASÉOLOGIQUE DU FRANÇAIS IVOIRIEN
}

\author{
Metáforas y Calcos en la CReación fraseológica del Francés de Costa de Marfil
}

Metaphors and caleues in French phraseological Creation in IVory Coast

\author{
Koffi Yao \\ Docteur Philologie Hispanique, \\ Université de Valladolid. Traducteur \\ et professeur département \\ d'espagnol, Université d'Abidjan \\ Cocody, Côte d'Ivoire. \\ Université Félix Houphouët-Boigny \\ de Cocody, 01 BPV 34 Abidjan 01, \\ Cocody, Côte d'Ivoire. \\ fyason@outlook.com
}

\begin{abstract}
RÉSUMÉ
L'objectif de cette étude est de mettre en évidence le principe qui régit la formation de certaines unités phraséologiques particulières du français parlé en Côte d'Ivoire, en s'appuyant sur l'utilisation de la métaphore et du calque lexico-sémantique en tant que procédés de reproduction du substrat linguistique local. Plus exactement, elle permet de cerner les propriétés idiomatiques qui caractérisent la formation des lexies complexes (expressions figées) et des lexies textuelles (adages ou proverbes) sélectionnées à partir de certains actes de communication courants entre les Ivoiriens. En outre, elle analyse l'influence des langues locales, en tenant compte des phénomènes contextuels ou socioculturelles environnants, à l'effet d'appréhender les principes théoriques qui déterminent la formation de ces unités phraséologiques spécifiques du français ivoirien.
\end{abstract}

Mots clés : création phraséologique, métaphores, calques, lexies textuelles, français ivoirien

\section{RESUMEN}

El presente estudio se propone poner en evidencia el principio que rige la formación de ciertas unidades fraseológicas específicas del francés hablado en Costa de Marfil, con base en el uso de la metáfora y el calco léxico-semántico en su calidad de procedimientos de reproducción del sustrato lingüístico local. Más precisamente, permite delimitar las propiedades idiomáticas características de la formación de lexías complejas (expresiones fijas) y de lexías textuales (adagios o proverbios) seleccionados a partir de ciertos actos de comunicación comunes entre los marfilenses. Por otro lado, analiza la influencia de las lenguas locales, teniendo en cuenta fenómenos contextuales o socioculturales circundantes, con el fin de aprehender los principios teóricos que determinan la formación de dichas unidades fraseológicas específicas del francés marfilense.

Palabras claves: creación fraseológica, metáforas, calcos, lexías textuales, francés de Costa de Marfil

\section{Abstract}

This study aims to shed light on the principle governing the formation of certain phraseological units specific to the French spoken in Côte d'Ivoire. Relying upon 
metaphors and lexical/semantic calques modeled on the local linguistic substrate, we can understand the idiomatic principles characterizing the formation of complex lexis (frozen expressions) and textual lexis (adages or proverbs) by relying on selected elements through some current speak acts between Ivorians. Otherwise, the study examines native language influences, taking into account the contextual and sociolinguistic phenomena, with the aim of pointing the theoretical properties determining the construction of those specific phraseological units of the French spoken in Ivory Coast.

Keywords: phraseological creation, metaphors, calques, textual lexies, Ivorian French 


\section{Introduction}

La variation polycentrique de la langue française est suffisamment démontrée dans plusieurs études scientifiques proposées par nombreux linguistes. Les phénomènes linguistiques et les preuves formelles ou structurelles que l'on pourrait fournir à cet effet sont abondants. À la base, il faut noter qu'au niveau lexico-sémantique les différences relevées sont profondes. Au-delà des emprunts obligatoires relevant de l'adoption ou l'adaptation du nom de certains objets exotiques dans chacune des variétés du français, nous pourrions citer quelques innovations expressives du français local comme : attraper le cour (être courageux), gâter la tête (inculquer de mauvaises habitudes à quelqu'un ou rendre fou), faire la bouche (faire la grande gueule ou être arrogant) etc., communément employées dans plusieurs pays de l'Afrique subsaharienne. Partant de ces exemples, on peut affirmer très clairement que le français en Afrique présente un ensemble d'éléments distinctifs particuliers qu'il conviendrait d'examiner de façon systématique. C'est d'ailleurs ce qui incite Matoré (1953, p. 14) à souligner « l'importance du facteur social dans les évolutions lexico-sémantiques; changements linguistiques portant sur les nuances sémantiques $\gg$. Nous essaierons de mettre en lumière toute cette problématique en nous appuyant sur l'analyse d'un corpus composé de Lexies Complexes et des Lexies Textuelles produites par les Ivoiriens. Aussi, conviendrait-il de relever tout l'intérêt que revêt cette initiative qui trouve sa justification non seulement dans la nouvelle perspective épistémologique qu'elle propose mais aussi dans sa contribution à l'étude du français ivoirien. En effet, il faut observer que chez les Ivoiriens, par exemple, lorsque quelqu'un « demande la route », cela ne signifie pas qu'il ne reconnait plus son chemin ou qu'il ne se retrouve plus, ou qu'il s'est égaré. En effet, par cette sollicitude, en respect des coutumes ou pratiques traditionnelles, ce dernier demande tout simplement l'autorisation et la bénédiction de son hôte pour prendre congé de lui. Dans ce cas, on dirait en français standard: demander à partir ou à se retirer. De même, on emploie faire le côcô $(\mathrm{du}$ baoulé), là où le français standard dirait : demander la main.

L'analyse structurelle de ces collocations permet d'indiquer qu'il s'agit ici d'un calque lexico-sémantique qui est la transposition d'une idée (sens) empruntée du substrat linguistique ivoirien pour l'adapter à la forme du français. On dénombre plusieurs expressions de ce genre qui, très souvent, entraînent des incompréhensions ou même des confusions entre certains interlocuteurs ${ }^{1}$. Partant de ce constat, nous avions réalisé une étude sur l'inventaire des particularités phraséologiques ou idiomatiques du français ivoirien ${ }^{2}$. À la suite de celle-ci, il nous a paru à la fois intéressant et nécessaire d'entreprendre cette analyse théorique qui permet de comprendre les principes généraux régissant la formation de ces unités verbales caractéristiques du français de Côte d'Ivoire. Plus spécifiquement, elle a pour objet d'expliquer le mode d'adaptation de cette langue exogène qui est le français aux réalités locales en s'appuyant sur des locutions et expressions proverbiales construites à partir de métaphores et de calques basées sur des réalités ou des usages courants entre les Ivoiriens. En d'autres termes, cette étude va s'appuyer sur un ensemble d'éléments lexico-sémantiques tant du français que des langues locales ivoiriennes pour essayer d'élucider la façon dont les locuteurs procèdent à la construction de ces locutions ou énoncés phraséologiques spécifiques qui permettent de représenter plus fidèlement les pratiques et la pensée ou encore la vision des Ivoiriens. Cela dit, il s'agira de comprendre le rapport entre la langue et la société tout à partir de l'influence des phénomènes psychologiques ou psycholinguistiques et des facteurs socio-culturels

1 Ce sont des pratiques linguistiques très usuelles entre les Ivoiriens mais, lorsque les interlocuteurs sont des étrangers ou des occidentaux, en particulier, très souvent ceux-ci ne comprennent pas spontanément le sens de ces expressions.

2 Particularités phraséologiques du français ivoirien: Lexies Complexes (LC) et Lexies Textuelles (LT) 
dans la conception typologique ou structurelle de ce parler régional que représente le français ivoirien.

\section{Aspects théoriques et méthodologiques}

La connaissance des concepts qui structurent cette étude et la méthode utilisée pour sa réalisation sont deux aspects fondamentaux à définir avant tout. D’abord, au niveau méthodologique, il faut préciser que nous avons procédé à la consultation documentaire et aussi à l'enquête expérimentale par l'observation directe des échanges entre les locuteurs pour constituer le corpus. Relativement à la présentation de ces données, nous analysons, d'une part, des calques du français ivoirien venant des langues nationales et d'autre part, des expressions du nouchi, argot populaire de Côte d'Ivoire, formé de l'hybridation d'éléments verbaux issus de certaines langues étrangères (français, anglais, espagnol, etc.) et du substrat linguistique local (baoulé, dioula, bété, etc). Il importe de noter que chaque item du corpus sera formellement défini et les origines ou les conditions de leur formation seront analysées.

Après ces précisions, nous nous occuperons de porter un éclairage sur les notions ou concepts essentiels antérieurement indiqués. Nous essaierons donc d'expliquer ce que l'on entend par français ivoirien et aussi les lexies complexes et textuelles qui constituent l'objet central de la réflexion que nous entreprenons.

\section{Le français ivoirien}

Contrairement au français central ou métropolitain perçu par le monde francophone comme la norme de référence, le français ivoirien, s'écartant structurellement de ladite norme, se présente comme un amalgame de parlers marqué par l'enchevêtrement de différentes formes d'usage adaptées aux besoins des usagers dont la complexité pose un souci de classification qui a suscité plusieurs réflexions. En effet, ses caractéristiques structurelles ne rendent nullement aisée l'étude sociolinguistique même si, a priori, il est évident de constater des variations diastratiques que l'on associe souvent à la compétence des locuteurs ou quelquefois à leur niveau intellectuel.

Quoi qu'il en soit, on comprendra que le français ivoirien présente des variations importantes dont la stratification paraît une épreuve laborieuse qui ne saurait donner une appréciation systématique. C'est pourquoi Boutin (2002) affirme qu'il est complexe de formuler une définition formelle à cet effet. Ainsi, s'appuyant sur un ensemble d'analyses fondamentales et pertinentes, cette linguiste indique que le français ivoirien pourrait bien s'associer à une multitude de notions théoriques telles que véhicularisation et vernaculisation, appropriation et variation, normes endogènes, etc. Suivant cet ensemble de concepts, on pourrait s'apercevoir de la multiformité de ce français qui fait l'objet de cette étude. Cependant, Lafage (2002), précise qu’à défaut d'une langue locale véhiculaire ou koiné, les Ivoiriens se sont appropriés la langue française pour satisfaire les besoins d'échange interethnique. Cette appropriation s'opère fondamentalement sur la base d'une adaptation structurelle qui est rendue possible grâce à la créativité verbale des locuteurs, montrant l'intérêt ou le souci d'accommoder le français au contexte ou aux réalités sociales. Cette préoccupation est aussi relevée par Duponchel (1975) qui souligne l'importance « des faits de création lexicale qui concerne le français de Côté d'Ivoire ».

Par ailleurs, Aboa (2008) observe l'existence d'une multitude d'usages et de modes d'appropriation de la langue française, influencée par le contexte de la pratique ou par la couche socioculturelle dont le locuteur fait partie. De ce point de vue, on peut identifier trois variétés du français qui correspondent systématiquement à différents niveaux de compétence linguistique.

On observe un net rapprochement entre les variétés mésolectale (français parlé par la classe moyenne) et acrolectale (français standard parlé par l'élite sociale) et une assimilation de certains traits des variétés ba- 
silectales (français parlé par la classe moyenne basse, composée de scolarisés de l'enseignement primaire, d'alphabétisés en français ou d'illettrés parlant français). Il existe donc des formes endogènes du français en Côte d'Ivoire qui tendent à s'uniformiser. Mais le fait le plus remarquable est que les Ivoiriens, dans leur pratique quotidienne, ont pris conscience du caractère spécifique de leur « français ». (Kouadio, 2008, p. 196)

En effet, cette stratification sociolinguistique permet de structurer les variantes de ce qu'on appelle le français ivoirien. Ce parler local présente des normes lexico-sémantiques et morphosyntaxiques très distinctives, $s$ 'imprégnant des réalités sociolinguistiques et socioculturelles locales. Des chercheurs comme Mauny (2011) et Aboa (2012) ont expliqué ou démontré que les différentes variétés du français ivoirien étaient la résultante d'un vif esprit créatif ivoirien et correspondaient à des besoins communicatifs spécifiques.

Ainsi, les expressions qui serviront à étayer notre argumentaire, attestent bien qu' « une langue n'est jamais dans un lieu et dans un groupe social donnés, identique [...] dans un autre lieu, dans un autre groupe social. » (Dubois et al., 2002, p. 504). Cette approche diasystémique de l'analyse linguistique concerne des changements qui interviennent dans tout système ou toute langue pendant son évolution. C'est ce principe fondamental qui est mis en évidence à travers les éléments linguistiques que nous proposons dans cette étude.

\section{L'unité phraséologique}

La phraséologie appartient au domaine de la lexicographie, et elle est l'objet plusieurs approches théoriques. D'une part, suivant une analyse conceptuelle proposée par Gledhill et Frath (2007), on désigne par ce terme, une combinaison syntagmatique obéissant aux contraintes du système lexico-grammatical. Techniquement, on l'appelle aussi collocation ou synthème selon Martinet (1974) et il peut s'appliquer à une expression déjà connue dans une langue donnée ou à une innovation expressive (créativité phraséologique) qui vient s'ajouter au lexique d'une langue. D'autre part, suivant une approche typologique, Pavel (1993) parle d'une unité fondée sur la nature et les relations du concept désigné par leur noyau terminologique.

Cette terminologie est aussi employée par Corpas (1996) pour désigner les phrases proverbiales ou lexies textuelles (LT) et les locutions courantes telles que les lexies complexes (LC). La lexie correspond, selon Pottier (1969), à l'unité lexicale ou le mot en tant que structure fondamentale du langage. Techniquement, cette désignation est beaucoup plus spécifique et présente les éléments lexicaux en termes de a) lexie simple, b) lexie composée, c) lexie complexe et d) lexie textuelle.

En général, ces formes lexicales ont été répertoriées dans des glossaires ${ }^{3}$ des particularités lexicales du français ivoirien. Cependant, il se posait la nécessité de traiter plus spécifiquement les expressions idiomatiques et proverbiales sur le plan théorique. Cette préoccupation impliquera nécessairement l'exploration de plusieurs approches définitionnelles du concept de phraséologie.

Rey (1970) indique que l'unité phraséologique est un système de particularités expressives liées aux conditions sociales dans lesquelles la langue est actualisée. Selon le Petit Robert (2016), il s'agit de groupes de mots formant une unité et ne pouvant être modifiés à volonté. Ainsi, par phraséologie, on entend les possibles combinaisons par lesquelles peuvent s'organiser les unités structurelles d'une langue. Pour sa part, Sevilla (2014, p. 7) relève que l'unité phraséologique est une construction pluriverbale composée de mots dont le nombre, la valeur et la fonction sont déterminés par une structure interne formant un ensemble cohésif. Cependant, nous notons que ces définitions sont très souvent génériques et ne mettent pas en évidence les propriétés basiques de ce que nous pouvons entendre par expressions phraséologiques, qui doivent nécessairement être déterminées par des concepts rigoureux.

3 Cf. Duponchel (1970), Équipe IFA (1988). 
De ce point de vue, la définition de Varela et Kubarth (1994) semble la plus appropriée, car elle s'appuie sur des critères syntagmatiques très pertinents : la stabilité et l'idiomaticité. Nous nous occuperons de porter un éclairage sur ces principes fondamentaux dans les sections suivantes.

\section{La stabilité}

Suivant l'approche structurale, le critère de stabilité est un principe essentiel de la formation de l'unité phraséologique: « hay unidad fraseológica alli donde se produce una secuencia de palabras que permanece fija o estable a través de un determinado periodo de tiempo » (Varela et Kubarth, 1994, p. 7).

Il est important de relever que la stabilité joue un rôle tout aussi important au niveau lexico-sémantique car l'idée exprimée ou le sens de l'expression dépend de l'unité syntagmatique dans son ensemble et de la typologie selon laquelle elle est structurée. Par exemple : « grand type » et « type grand » ne signifient pas la même chose. Cela dit, l'unité idiomatique doit absolument obéir à sa structure stéréotypée afin de conserver son sens. La moindre modification subie est susceptible d'entrainer sa déstructuration typologique et aussi sémantique. Par exemple, une expression comme « demander/donner les nouvelles » enregistrait des changements formels et sémantiques importants si un seul composant venait à être modifié, comme dans « demander/donner quelques nouvelles ». Dans ce cas, cette unité expressive ne garderait plus sa cohésion formelle en tant que locution verbale et perdrait aussi son sens particulier qui est celui de s'enquérir des motifs d'une visite.

\section{L'idiomaticité}

Selon Dubois et al. (1994), «se llama idiomatismo a toda construcción perteneciente a una lengua determinaday que noposeeninguna correspondencia sintáctica en otra lengua ». Ainsi, l'idiomaticité fait allusion aux constructions stéréotypées et spécifiques à une langue donnée. Ce principe est aussi déterminé par le critère de l'inséparabilité ou la stabilité des composantes et par la cession totale ou partielle des éléments sémiques de chacune de ces unités qui interviennent dans l'ensemble de la structure syntagmatique.

Par ailleurs, il serait tout aussi intéressant de relever l'importance de la sélection des éléments verbaux et de leur association particulière dans la formation des expressions idiomatiques de chaque langue. Ceci est un processus correspondant à un concept, c'est-à-dire un ensemble d'idées ou un mode d'appréciation intellectuelle s'appuyant sur l'échelle des valeurs des locuteurs. C'est ainsi la raison pour laquelle, sur le plan socioculturel, on admet certaines phrases ou certaines expressions comme actes du discours spécialisé ou simplement comme forme d'expression à valeur rituelle. Ces combinaisons à usage commun sont considérées comme un mot ou une unité verbale lorsque la communauté ou le groupe social que représentent les locuteurs l'admettent et le reconnaissent comme une structure lexicale correcte dans les usages conventionnels. Ainsi, à chaque culture correspondent des formules spécifiques de communication basées généralement sur des proverbes qui représentent un moyen stylistique dans la communication socioculturelle. Pour le cas d'espèce, nous essaierons de dégager les empreintes du vécu quotidien des Ivoiriens dans leurs usages particuliers de la langue française. Mais avant, il conviendrait de définir les termes LC et LT dans les chapitres suivants.

\section{Principes de formation de la lexie complexe (LC)}

La LC est une unité fonctionnelle composée d'un groupe de mots correspondant à un ensemble lexicalisé que l'on désigne aussi par le terme locution ou expression. Pour Tournier (1991), elle est une unité lexicale mémorisée sans limite de longueur, telles les citations et les expressions idiomatiques. La LC équivaut à un constituant compris entre le syntagme (exclusivement) et la phrase simple (exclusivement). Ainsi, on trouvera dans le français ivoirien des exemples comme: « chercher la bouche de quelqu'un »: provoquer quelqu'un; 
« casser le coup de quelqu'un »: éconduire quelqu'un; « manger son piment dans la bouche de quelqu'un » : être peu courageux ou être lâche, etc.

Aussi faut-il indiquer que ces unités lexicales particulières sont le résultat d'un processus de lexicalisation (cfr. Yao, 1998) ayant atteint un important niveau de cohésion ou d'homogénéité. Même si certains spécialistes comme Varela et Kubarth (1994) s'y réfèrent comme des unités en voie de lexicalisation, il s'agit essentiellement d'un phénomène de combinaison morphologique et sémantique par lequel un ensemble de morphèmes formant un syntagme se transforme en une unité lexicale formellement figée. Cela implique systématiquement la fusion totale ou partielle de tous les éléments morphématiques en vue de représenter une idée ou un concept. Au regard de ce constat, ce processus pourrait être perçu comme une $\ll$ relexicalisation ».

\section{Principes de formation des lexies textuelles (LT)}

La LT équivaut à une LC présentant les caractéristiques d'un texte. Il s'agit, plus précisément d'une unité syntagmatique qui atteint le niveau d'un énoncé $e^{4}$. Tenant compte des caractéristiques structurelles et sémantiques, on peut considérer la LC comme un segment (dépendant) du texte et la LT comme un texte complet et indépendant. Elle désigne un proverbe, un discours ou un adage. Exemple: « Yeux connait bagage qui est lourd, c'est bêla qui fait exprès $\gg$ : il faut être humble ou ne pas être prétentieux.

$\mathrm{Du}$ point de vue théorique, l'étude de la LT n'appartient pas au domaine de la phraséologie. Cependant, on peut s'apercevoir que la LT et la LC présentent d'énormes similitudes, c'està-dire qu'elles partagent les mêmes principes structurels et sémantiques, sans différences fondamentales. C'est en raison de ces facteurs que nous

4 Cfr. Corpas Pastor (1996) qui utilise le terme énoncé phraséologique pour désigner le proverbe établissons ce rapprochement qui permet de les présenter conjointement dans cette étude.

En outre, il est important d'indiquer que les données qui sont proposées proviennent généralement des variétés basilectale ou mésolectale, mais on peut aussi les retrouver dans les variantes comme le nouchi et quelquefois dans la forme acrolectale. Ces locutions ou énoncés phraséologiques très utilisés dans les échanges interpersonnels en raison de leur caractère expressif, c'est-à-dire leur capacité à représenter des idées spécifiques et à transmettre des messages assez clairs et précis, sont généralement utilisés dans tous les registres, en dépit de leur spécificité et des critères déterminant leur emploi. En outre, il conviendrait d'indiquer que de nos jours les variétés acrolectale et mésoletctale $\mathrm{du}$ français ivoirien sont quasiment confondues, alors que le nouchi a fini par absorber le français populaire.

\section{L'apport du substrat linguistique local dans la formation des LC et des LT}

Il convient d'indiquer que nous nous situons dans un contexte de contact de langues qui met en rapport des langues locales et le français. Celui-ci exerce une influence systématique sur la lexicogenèse ou la formation des unités de signification. Cet environnement sociolinguistique va engendrer la formation de diverses expressions amalgamées ainsi que des calques morphologiques et sémantiques. Cela dit, la majorité desdits éléments (LC et LT) présente certaines particularités structurelles et sémantiques qui relèvent de l'incidence du substrat linguistique local.

En outre, il faut expliquer que le terme « expression amalgamée » désigne une unité hétéroclite, dont les composantes morphématiques sont issues du français et de certaines langues locales. Exemples: faire le côcô, faire anago-plan, etc. Quant au calque morphologique ou sémantique, il représente un mécanisme de lexicogenèse permettant 
de désigner une pratique socioculturelle ou des notions inexistantes chez les Ivoiriens et pour lequel il faut obligatoirement trouver les expressions équivalentes du français qui sont susceptibles de les exprimer correctement (voir exemples antérieurs: « demander la route » [demander la permission à son hôte], « chercher la bouche de quelqu'un $\gg$ [provoquer quelqu'un], etc.)

\section{Fonctionnement de la métaphore et du calque}

Le dynamisme des variantes du français de Côte d'Ivoire est manifeste dans l'importante activité inventive dont l'objectif est de satisfaire tant le vide linguistique que d'adapter l'outil de communication aux besoins des locuteurs. Cette expérience qui implique, par ailleurs, la création des néologies ou des unités lexico-grammaticales à travers la métaphore et le calque, nécessite également une analyse constante. En général, ces éléments verbaux qui s'imprègnent de l'expérience et du mode de pensée des Ivoiriens sont entièrement fossilisés dans la mémoire collective et dans le parler de ces locuteurs.

Aussi, faut-il rappeler que la majorité des locutions et expressions proverbiales proposées proviennent fondamentalement de la littérature traditionnelle (contes et chants) et du nouchi (argot populaire de Côte d'Ivoire) (cfr. Yao, 2013) ou, quelquefois, des genres musicaux modernes (le zouglou et le coupé-décalé). Nous essaierons d'expliquer ces phénomènes linguistiques à travers quelques exemples qui apparaissent dans les chapitres suivants.

\section{Lexies Complexes empruntées des langues nationales}

\section{Affaire $n f:$ Chercher/mettre/donner une affaire \\ Le mot affaire sert à construire diverses expressions idiomatiques dans le français ivoirien. En général, il est accompagné d'un verbe tel que: chercher, mettre, donner, etc. En réalité, la majorité des}

expressions employées dans cette étude existent déjà dans certaines langues locales (agni, baoulé, dioula, etc.) et c'est à partir de celles-ci que les locuteurs reproduisent les calques qui composent le glossaire que nous proposons. Par exemple, le mot affaire se dit en baoulé ndè et ko en dioula. Ces substantifs sont respectivement accompagnés des verbes génériques comme koundèlè du baoulé ou gnili du dioula pour construire les expressions ndè koundèlè ou ko gnili, correspondant à la lexie complexe représentée dans le français ivoirien: chercher affaire.

Chercher affaire : provoquer ou chercher noise. Exemple : Il ne faut pas chercher affaire où il n'y en a pas.

Mettre affaire (sur quelqu'un) : accuser quelqu'un. Exemple: Ce n'est pas sur moi que vous allez mettre votre affaire. Je n'étais pas présent au moment des faits.

Donner affaire à quelqu'un : mettre quelqu'un dans des problèmes. Exemple: C'est toi Yao qui donne toujours affaire aux gens. Cela ne peut pas continuer ainsi.

Nous trouverons aussi diverses expressions idiomatiques qui se construisent à partir de certains éléments somatiques comme la bouche. Il s'agit également de calques lexico-sémantiques basés sur certaines langues locales. Voyons les exemples suivants:

Avoir la bouche sucrée : être rusé, savoir convaincre. Exemple : C'est avec sa bouche sucrée qu'il a réussi à convaincre cette fille de l'épouser.

Mettre la bouche dans une affaire : s'impliquer dans une affaire. Exemple: Un enfant ne doit pas mettre la bouche dans les affaires de ses ainés.

Faire la bouche : avoir la grande gueule. Exemple: Jean fait la bouche. Pourtant il est le plus paresseux de tous. 
Enlever la bouche sur quelqu'un : laisser quelqu'un en paix. Ne pas déranger quelqu'un. Exemple : Je ne suis pas d'humeur ce matin, alors enlève ta bouche sur moi.

Attacher la bouche de quelqu'un : clouer le bec à quelqu'un. Exemple : il ne s'est pas défendu ; c'était comme si on lui avait attaché la bouche. Voir aussi : Manger son piment dans bouche de quelqu'un (LT)

Demander/donner les nouvelles : s'enquérir des raisons de la visite d'une personne / donner ou expliquer les raisons de sa présence. Exemple : vous vous êtes bien reposés? À présent, nous vous demandons les nouvelles. Cette expression est très courante chez les baoulés qui disent : djasin tilè. Djasin signifie la nouvelle que l'on apporte et tilè est un verbe générique qui signifie ici s'enquérir de. Ainsi, suivant les us et coutumes, elle s'applique à la formalité consistant à s'informer des nouvelles de l'autre, ce qui symbolise le partage. Ainsi, quand on rencontre quelqu'un ou qu'on rend visite à quelqu'un, on doit donner les nouvelles. Cette pratique implique dans une certaine mesure le souci du partage ou de la convivialité chez les Ivoiriens et les Africains en général.

Faire atou : donner des accolades. Exemple : Viens me faire atou, mon petit-fils, ça fait une éternité que nous ne nous sommes pas vus. Il faut noter que le mot atou vient du baoulé et signifie faire des accolades ou prendre quelqu'un dans ses bras (en lui souhaitant la bienvenue) ou encore faire des compliments à quelqu'un.

Faire le côcố : se présenter officiellement à des beaux-parents. Exemple : Après avoir passé tant d'années avec cette fille, je crois qu'il est temps d'aller faire le côcô. Il est important de relever que le mot côcô vient du baoulé et qu'il représente une onomatopée correspondant à toc-toc en français. Il est employé dans cette expression pour dire qu'on frappe à la porte afin de se présenter aux beaux-parents en officialisant, à la fois, les relations avec leur fille. En outre, faire le côcô est un rituel hautement symbolique et important chez les akans. Il précède le mariage coutumier ou traditionnel et le mariage administratif ou officiel.

Lancer foulard : exercer la prostitution. Le foulard est un accessoire vestimentaire très apprécié par les femmes africaines qui l'utilisent comme un ornement pour protéger les cheveux. Elles l'attachent quelquefois à la ceinture. Le lancer viendrait à se donner ou, à tout le moins, à donner une partie d'elles à une tierce personne. Traditionnellement, lancer ou jeter le foulard sur quelqu'un est aussi une pratique par laquelle on invite souvent des personnes à se joindre à une danse sur la place publique. Ce rituel a été adopté par les prostituées ivoiriennes pour aguicher les potentiels clients. Exemple : Dans certains quartiers de la capitale, des filles lancent foulard.

Mari de nuit, femme de nuit : esprit incube / succube. Cette expression calquée du baoulé relève de la croyance populaire selon laquelle un homme ou une femme se sent fréquemment soumis à des relations sexuelles avec des esprits ou des êtres de l'au-delà. Exemple : Nous allons voir un marabout pour chasser ton mari / ta femme de nuit.

Mettre/verser du sable dans l'attiéké de quelqu'un : empêcher quelqu'un de s'épanouir ou de réussir. L'attiéké est un plat local très prisé par la population ivoirienne et, ainsi, il représente une substance essentielle symbolisant, par la même occasion, la (sur)vie. Par conséquent, y jeter du sable équivaudrait à la destruction ou l'empêchement d'être épanoui ou d'être heureux. Exemple : J'ai travaillé très dur pour réussir dans la vie, alors ne verse pas du sable dans mon attiéké.

Venir à Abidjan pour regarder la lagune : dormir sur ses lauriers. Exemple : $\mathrm{Si}$ tu es venu à 
Abidjan pour regarder la lagune, tu retourneras malheureux d'où tu viens.

Verser de l'eau : faire une libation dans le but de bénir ou maudire. 1. Maudire. Exemple: Après avoir commis cette énorme bêtise ses parents ont versé de l'eau. Ils l'ont maudit. 2. Bénir. Exemple : Avant le début de la plupart des cérémonies nationales et solennelles, la chefferie hôte de ladite cérémonie verse de l'eau pour que tout ce passe bien.

\section{Lexies Complexes empruntées au nouchi}

C'est baby c'est loto : imbroglio, confusion. Cette expression vient de la juxtaposition de deux phrases dont la signification s'appuie sur les attributs, c'est-à-dire l'éponyme baby (modèle appelé deux chevaux de la marque Citroën) et l'hypéronyme loto (dérivé du mot automobile qui s'emploie dans certaines langues locales) pour créer un pléonasme désignant une situa-

Drap : ce mot drap du français central qui désigne une toile a expérimenté un changement sémantique dans le nouchi. Il est polysémique et acquiert une signification spécifique selon l'expression ou le contexte de son emploi.

Être en drap : 1 . Informer ; 2. Connaître, savoir ou prendre conscience de quelque chose. Exemple: Mes amis sont en drap de tout ce que je fais.

Être enjaillé de quelque chose : aimer, apprécier. Le mot enjailler est une néologie de l'anglais enjoy que le nouchi a adopté en l'adaptant au français qui est sa structure de base. Cette expression peut s'appliquer à la musique (exemple : je suis enjaillé du zouk) ou à un évènement. Exemple : Paul est enjaillé par la démarche de Marie. C'est aussi de cette création lexicale que dérive l'expression enjaillement dans ses dents qui signifie être éperdument amoureux.

Mettre en drap ou prendre drap : humilier. Syn. Mettre soua

Dja foule : 1 . Tancer ou sermonner quelqu'un ; 2 . Amuser ou égayer. Il s'agit d'une structure lexico-grammaticale formée de la combinaison du mot dja, une néologie nouchi qui signifie tuer, et foule qui signifie multitude ou abondance. Cette expression a une valeur emphatique et connote l'exagération.

\section{Lexies textuelles empruntées des langues nationales}

Cabri mort n'a pas peur de couteau : s'en foutre. On emploie cette expression lorsqu'on est face à l'adversité et qu'on sent qu'on n'a plus rien à perdre, ou lorsque la situation est déjà hors de contrôle, mais qu'il faut y aller sans vraiment se préoccuper de ce qui pourrait arriver. Dans certains rituels traditionnels, le cabri est souvent donné en sacrifice pour exorciser le mal. Il symbolise chez les Ivoiriens la vie ou la mort et aussi l'insouciance face au danger. C'est à partir de cette métaphore qu'est née l'expression ci-dessus indiquée.

Ce sont les deux mains qui se lavent : l'union fait la force. En général, les deux mains mises ensemble symbolisent l'entraide et l'union. Ainsi, on est plus fort quand on se met ensemble pour entreprendre une action. C'est-à-dire qu'on réussit toujours en agissant en synergie. En effet, en lavant (quelque chose) avec les deux mains, on obtient de meilleurs résultats que lorsqu'on le fait avec une seule. Dans la même perspective, on dit aussi : «C'est l'homme qui fait l'homme », pour dire qu'on a toujours besoin de se faire aider pour réussir.

Découragement n'est pas ivoirien : les Ivoiriens sont vaillants et ne baissent jamais les bras. Cette expression qui traduit la résistance face aux difficultés est un moyen pour encourager et montrer l'esprit combatif du peuple ivoirien. Cette citation du Président Houphouët Boigny a fini par se consolider et s'établir comme une expression figée du français ivoirien. 
Dent pourrie est mieux que bouche vide : il faut se contenter de peu ou de ce qu'on a. Équivaut à « un tien vaut mieux que deux tu l'auras ». On dit aussi : un est mieux que zéro. Cet adage qui est tiré de la sagesse populaire et repris dans le français local, recommande que l'on sache valoriser et accepter ce dont on dispose sans envier autrui. Il est souvent employé dans des situations où l'on estime avoir obtenu un mauvais résultat ou être lésé.

Derrière village il y a village : rien n'est absolu. Le village est un symbole important pour les Ivoiriens en ce sens qu'il représente leurs racines, leurs traditions ou encore le lieu de formation ou de réalisation culturelle et identitaire. Cette locution proverbiale exprime dans l'entendement des ivoiriens le dynamisme naturel et l'évolution perpétuelle qui caractérise chaque chose de la vie.

\section{L'éléphant va beau maigrir il ne rentrera jamais} dans poulailler : on reste toujours ce qu'on est. L'éléphant, symbole national de la Côte d'Ivoire, est un animal fort et puissant et d'une grande envergure. Ainsi, dans l'imaginaire des Ivoiriens, quelles que soient les difficultés, il restera toujours fort, et on lui reconnaitra toujours sa nature en terme de puissance et de pouvoir.

La main qui demande est toujours en bas : lorsqu'on est dans le besoin il faut se montrer humble. Baisser la main lorsqu'on est dans le besoin est signe d'humilité et de sagesse. Cet adage du français ivoirien exprime inversement l'adage en français standard « la main qui donne est celle qui ordonne $\gg$.

Le mouton broute là où on l'attache : il faut s'adapter aux conditions qui s'imposent à soi. Cet adage explique que parfois la solution aux difficultés se trouve si près, c'est-à-dire que les opportunités sont près de nous et qu'il ne faut pas les chercher ailleurs ou plus loin.
Les moutons se promènent ensemble mais ils n'ont pas les mêmes prix : chaque individu a sa propre valeur, ses qualités et ses défauts. Ce proverbe relève les différences entre les hommes. Quand bien même nous pouvons avoir quelques traits communs, nous ne serons jamais identiques au sens strict du terme. Dans la culture ivoirienne, le mouton est généralement pris comme l'animal approprié pour faire des sacrifices. Il symbolise aussi la différence de valeur entre les individus. Ainsi, à l'image du mouton que l'on valorise par ses caractéristiques (taille, couleur, etc.), chaque personne a sa particularité qu'il convient de considérer.

Lorsque le tonnerre gronde, chacun attrape sa tête : chacun pour soi et Dieu pour tous. Cela signifie que l'être humain est foncièrement égocentrique. Si bien que dans les circonstances difficiles, il pense à lui-même avant de s'intéresser à la situation des autres. Dans l'imaginaire des Ivoiriens, le tonnerre entraîne nécessairement la foudre qui symbolise le danger ou la difficulté. Face à ces circonstances, on attrape la tête, c'est-à-dire qu'on se protège soi-même pour préserver la vie.

L'étranger a de grands yeux mais il ne voit rien : Quelle que soit la perspicacité d'un étranger, il ne peut pas percer tous les secrets. À cet effet, il est incapable de tout savoir sans qu'il en soit instruit. Ce proverbe qui est tiré de la sagesse populaire implique l'humilité, le respect de l'étranger envers son hôte qui « lui ouvrira les yeux $\gg$.

Manger son piment dans la bouche de quelqu'un : être lâche ou poltron. Il faut dire que le piment est un condiment très apprécié par les Ivoiriens. Il est caractérisé par un goût piquant et brûlant. Au plan stylistique, ce proverbe relève d'une métaphore basée sur les attributs du piment. Ainsi, par comparaison, elle implique le refus de prendre ses responsabilités en se servant d'une autre personne pour 
résoudre des problèmes délicats et difficiles à assumer. On l'emploie généralement dans des phrases négatives. Exemple: « Ce n’est pas dans ma bouche que tu vas manger ton piment. »

On ne prend pas la moitié de cola pour faire un sacrifice : Pour rendre justice, il est impératif d'entendre toutes les parties belligérantes. Le cola est un fruit très prisé et d'une valeur hautement symbolique chez la majorité des peuples ivoiriens qui l'utilisent dans certains rituels traditionnels. Il représente l'union et sert à célébrer le mariage, la réconciliation, la paix, etc. Ce proverbe qui s'inspire du cola relève d'une métaphore basée sur la morphologie, c'est-àdire la forme entrelacée des deux parties de cette noix.

On ne montre pas son village avec sa main gauche : Il faut ne faut pas mépriser son village. Il faut toujours être fier de ses origines. Cet adage traduit la représentation de la main gauche dans l'imaginaire des Ivoiriens. Celle-ci est considérée comme une mauvaise main. Ainsi, montrer son village avec cette main signifie qu'on le méprise.

On ne reste pas dans magnans pour enlever magnans : Il faut prendre du recul vis-à-vis des problèmes afin de mieux les résoudre. On trouve très souvent des files de magnan sur le chemin des champs ou dans les champs. Le magnan est une espèce de fourmi très agressive et symbolise des problèmes auxquels les paysans ivoiriens sont confrontés quotidiennement. L'expérience montre qu'on n'arrive jamais à s'en débarrasser tant qu'on reste à l'endroit où ils se trouvent. C'est de là que vient ce proverbe qui, par métaphore, indique comment il faut s'y prendre face aux difficultés.

On ne regarde pas dans la bouche de celui qui grille des arachides : Il y a certaines choses qui sont inévitables et doivent être acceptées comme une fatalité. On ne peut pas faire grand-chose face à certaines situations, car elles s'imposent et on les subit (malgré nous). L'homme n'est pas toujours maître de ses propres circonstances, c'est-à-dire que nous n'avons pas toujours le contrôle de toutes les choses. En outre, faut-il retenir que l'arachide est beaucoup consommée par les Ivoiriens qui s'en servent pour faire des sauces (appelées communément : sauce-arachide) ou des cacahuètes, après torréfaction. Pour obtenir la qualité désirée, le grilleur doit en gouter à chaque moment pour contrôler le processus. Cette expérience qui, par métaphore, a donné cet adage qui se retrouve dans le parler courant des Ivoiriens est en fait une citation du Président Houphouët Boigny traduite du baoulé.

\section{On travaille pour payer mouton et non pour} devenir mouton : Nul ne doit se soumettre ou permettre qu'on abuse de lui. Le mouton a de la valeur et de l'estime dans certaines religions locales. En revanche, il est également considéré dans l'imaginaire collectif comme un animal moins intelligent. Ce proverbe met alors en contraste cette ambivalence de cet animal qui se transpose dans le fonctionnement de la vie sociale : payer mouton (avoir de la valeur financière et/ou sociale) et devenir mouton (faire preuve d'inintelligence/manquer de sagesse).

Quand la tête est là, le genou ne porte pas le chapeau : Il faut respecter la hiérarchie sociale. D'un point de vue anatomique, la tête se trouve dans une position supérieure par rapport aux autres parties du corps. C'est cette distribution de la morphologie humaine qui est métaphoriquement transposée dans la caractérisation pyramidale de l'organisation sociale de la majorité des peuples de Côte d'Ivoire où il y a toujours un ordre à respecter. En outre, il faut noter que cette locution proverbiale appartenant au groupe akan (groupe social très structuré et hiérarchisé) correspond à un calque lexico-sémantique.

Quand on t'envoie, il faut savoir t'envoyer : Il faut être sage et prudent dans l'accomplissement 
d'une mission. Ce proverbe ou phrase axiomatique est calqué du baoulé. Il faut en retenir que toutes les responsabilités portent en elles des risques. Alors, toute personne chargée d'une mission devrait être vigilante. Autrement dit, elle risquerait de s'attirer des ennuis ou de se retrouver dans des problèmes.

Relation est mieux que diplôme : Les diplômes ne suffisent aucunement pour atteindre ses objectifs. Il faut surtout s'appuyer sur quelqu'un qui peut vous donner un coup de main. Cette locution proverbiale qui relève l'importance des relations humaines pour la promotion socio-professionnelle décrit aussi la corruption et l'esprit d'éternel assisté qui caractérise les Ivoiriens.

\section{Si tu cherches ma bouche, tu vas croiser mes} dents : Qui cherche trouve. Correspond aussi au proverbe : qui s'y frotte s'y pique. (Voir bouche)

Sécher son linge là où le soleil brille : être opportuniste. Savoir saisir la bonne occasion au bon moment. Cette expression qui fait aussi allusion à la déloyauté a généralement une connotation péjorative.

\section{Lexies textuelles empruntées du nouchi}

Connaisseur connaît, gaou passe : L'individu qui répète les mêmes erreurs est stupide. Cette phrase proverbiale qui est désormais figée dans le français ivoirien vient du titre « Premier gaou n'est pas gaou »du groupe Magic System. Le mot gaou, qui est une néologie du nouchi, signifie: 1. Démodé; 2. Ignorant ou stupide; et s'oppose à connaisseur qui désigne une personne expérimentée et moderne.

Gbê est mieux que drap : Dire la vérité permet d'éviter l'bumiliation; équivalant aussi à: la vérité rougit les yeux mais ne les cassent pas. Ce proverbe est construit à partir du mot gbê: blanc en dioula, couleur qui symbolise la vérité et drap qui est une néologie du nouchi qui signifie : 1. Humilier; 2. Informer. Le mot $g b \hat{e}$ est souvent utilisé dans certaines combinaisons lexico-grammaticales avec d'autres éléments du français pour former des lexies textuelles : Trop parler donne dagbê : Mieux vaut agir queparler. Cette expression proverbiale est une construction verbale ou lexico-grammaticale propre au français ivoirien. Elle regroupe certains éléments du français standard et le mot dagbê ( $d a$ signifie bouche et $g b \hat{e}$, blanc) qui désigne les filets de bave à la commissure des lèvres.

Zyeux connait bagage qui est lourd : Chacun connaît ses forces et ses limites. Ce proverbe est inspiré de la pénibilité du travail des bêla ou porteurs de bagage dans des carrioles appelées aussi pousse-pousse par les Ivoiriens. L'analyse structurelle de cette construction indique qu' il s'agit d'une phrase composée d'un ensemble d'éléments du français standard et du mot zyeux (cette forme nominale du nouchi est toujours au pluriel, même si le verbe ne s'accorde pas).

\section{Discussion}

Les nombreuses oppositions à l'usage de ces expressions du français ivoirien de la part des défenseurs du bon usage de la langue française n’ont nullement freiné la poussée de ce phénomène d'adaptation ou de création verbale qui, bien au contraire, s'est imposé et répandu au point d'absorber presque tous les registres ${ }^{5}$, donnant ainsi une identité personnelle au français ivoirien. Au niveau lexico-grammatical, on retrouve

5 Notons que, pour des raisons stylistiques, même les détracteurs les plus critiques, comme c'est le cas de certains universitaires, ont fini par admettre l'importance sociolinguistique du français ivoirien et l'expressivité ou la pertinence sémantique de ces locutions et proverbes qu'ils utilisent souvent. Et, comme nous l'avons indiqué, l'évolution ou la transformation diachronique de cette variété du français a entraîné aussi la confusion des formes acrolectale et mésolectale, pendant que la forme basilectale est absorbée par le nouchi. 
souvent des coïncidences singulières entre le calque phraséologique et la forme du substrat, par exemple « demander la route » (man atin en baoulé). Mais aussi, on s'aperçoit que généralement, ces unités ne présentent aucune relation systématique avec les éléments originaux, ou du moins, leur rapport n'est pas formellement établi. Par exemple, « demander des nouvelles » (bisa jasin) qui signifie : demander ce qui précède le pied (nouvelles). Cette réalité confirme bien l'hypothèse de Gledhill et Frath (2007) qui soutiennent que toute construction nouvelle en phraséologie est le fruit d'une négociation au sein d'une communauté.

En outre, il est important de souligner que la différence entre deux systèmes lexico-grammaticaux résulte de l'idiomaticité des expressions en question. Leur interprétation sémantique relève d'un simple rapprochement qui tient compte de la valeur conventionnelle de la langue, tandis que les adaptations structurelles obéissent à un souci d'appropriation linguistique. Enfin, rappelons aussi que suivant son fondement théorique, l'unité phraséologique ou synthème correspond à la formation ou la création lexicale dans laquelle, selon notre analyse, les notions ou valeurs socioculturelles qui imprègnent la majorité de ces constructions apparaissent souvent comme un critère pertinent et essentiel qu'il conviendrait d'évaluer.

\section{Conclusion}

Il aurait probablement suffit d'utiliser une poignée d'éléments pour expliquer le principe de formation des LC et des LT sur la base de métaphores et de calques lexico-sémantiques, mais la pertinence de l'étude exigeait autant de données pour étayer notre hypothèse.

Ainsi, l'approche théorique de ce travail nous a permis d'explorer des principes généraux structurant les unités ou énoncés phraséologiques. À l'issue de cette analyse, il semble important de relever l'impact du facteur social ou contextuel dans la structuration de ces unités verbales. En effet, au regard des particularités lexicales enregistrées, nous nous apercevons qu'en dépit des controverses, il est nécessaire d'admettre que les langues, comme c'est le cas ici, tendent généralement à exploiter les facteurs environnementaux ou contextuels ou à s'en imprégner pour exprimer les besoins communicatifs des locuteurs. Cette hypothèse nous paraît désormais indiscutable. Cela dit, les éléments enregistrés se construisent sur la base de l'expérience ou des conditions de leur production. C'est pourquoi nous estimons que pour les besoins épistémologiques ou scientifiques, il conviendrait de redéfinir les principes théoriques auxquels il serait certainement fondé d'associer le critère socioculturel exprimé à travers l'usage des moyens stylistiques ou linguistiques antérieurement indiqués. Au demeurant, comme nous avons pu constater, on enregistre de nombreuses expressions ou locutions construites à partir de métaphores et de calques lexico-sémantiques et ce processus linguistique a permis de mettre en évidence le rapport entre l'objet ou la perception de celle-ci et sa représentation verbale (néologie). À ce titre, il semble intéressant de rappeler l'adage : venir à Abidjan pour regarder la lagune. Nous avions expliqué que la lagune d'Abidjan ou encore la lagune ébrié (par allusion aux autochtones) longe tout le littoral et, par son envergure ou son importance, représente un symbole significatif chez les Ivoiriens. Aussi, faisant référence à cette vaste étendue d'eau, on a fini par attribuer à la ville d'Abidjan le nom de perle des lagunes. C'est cet ensemble de valeurs qui a donné l'expression : venir à Abidjan pour regarder la lagune qui veut dire rester oisif (en passant le temps à contempler cette lagune qui entoure Abidjan, la capitale de la Côte d' Ivoire). Dans cet ordre, nous avions présenté plusieurs expressions et locutions proverbiales qui s'appuient sur ces facteurs extralinguistiques. (Voir : arachides, attiéké, bêla, etc.)

In fine, on pourrait s'apercevoir très clairement de l'usage de la métaphore et du calque 
lexico-sémantique dans la représentation verbale de certains phénomènes contextuels ou socioculturels. Aussi, conviendrait-il de réitérer que ces procédés de reproduction ou de création lexicale apparaissent comme des instruments importants qui permettent efficacement de rendre compte du contexte sociolinguistique et de l'ensemble des phénomènes extralinguistiques. Par conséquent, ils devraient constituer des principes fondamentaux à considérer parmi les critères qui déterminent la formation des unités phraséologiques de façon générale.

\section{Références}

Aboa Abia, A. L. (2008). «La francophonie ivoirienne. Enjeux politiques et socioculturels». Dans Documents pour l'histoire du français langue étrangère ou seconde. Acte du colloque tenu à l'Université de Versailles, Saint Quentin-en-Yveline, 40/41, 161-178.

Aboa Abia, A. L. (2012). «Le français en contexte urbain en Côte d'Ivoire». Sudlangues, 18, 13.

Boutin Akissi, B. (2002). Description de la variation: étude transformationnelle des phrases du français de Côte d'Ivoire. Thèse doctorale soutenue à l'université de Grenoble III.

Corpas Pastor, G. (1996). Manual de fraseología española. Madrid : Gredos.

Dubois, J. et al. (1994). Diccionario de Lingüistica. Madrid: Alianza.

Dubois, J. et al. (2002). Dictionnaire de linguistique. Paris : Larousse.

Équipe IFA (1988). Inventaire des particularités lexicales $d u$ français en Afrique noire. Paris : Edicef.

Duponchel, L. (1975). Dictionnaire du français de Côte d'Ivoire. Abidjan : Institut de Linguistique Appliquée.

Duponchel, L. (1970). Note sur Ies groupes consonantiques en cbrie et en alladian. A. Univ. Abidjan Linguist. 3(1), 31-45.
Gledhill, C. et Frath, P. (2007). «Collocation, phrasème, dénomination: vers une théorie de la créativité phraséologique». La linguistique, 1(43), 63-88, Presses Universitaires de France.

Kouadio, N. J. (2008). «Le français en Côte d'Ivoire : de l'imposition à l'appropriation décomplexée d'une langue exogène». Dans Documents pour l'histoire $d u$ français langue étrangère ou seconde, Acte du colloque tenu à l'Université de Versailles, Saint Quentin-en-Yveline, 40-41, 179-197.

Lafage, S. (2002). «Le lexique du français de Côte d' Ivoire. Appropriation et créativité». Dans Le Français en Afrique (Revue du Réseau des Observatoires du Français contemporain en Afrique), 16-17(2), Nice, CNRS/Institut de Linguistique Française.

Le Petit Robert (2016). Le petit Robert. Paris : Éditions Le Robert.

Martinet, A. (1974). Elementos de lingüistica general. Madrid : Gredos.

Matoré, G. (1953). La méthode en lexicologie. Domaine du français. Paris : Didier.

Mauny, R. (2011). Glossaire des expressions et termes locaux de l'ouest africain. Paris : OIF, Écriture.

Pavel, S. (1993). «Vers une méthode de recherche phraséologique en langue de spécialité». Dans L'actualité terminologique/Terminology update, 26(2), 9-13.

Pottier, B. (1969). Introduction à l'étude des structures fondamentales. Nancy : Faculté des Lettres et Sciences Humaines.

Rey, A. (1970). La lexicologie. Paris: Klincksiek.

Sevilla M., J. (2014). Fraseología y léxico. Un enfoque contrastivo. Lugo : Axac.

Tournier, J. (1991). Introduction descriptive à la lexicogénétique de l'anglais contemporain. Paris : Champion Slaktine.

Varela, F. et Kubarth, H. (1994). Diccionario fraseológico del español moderno. Madrid: Gredos.

Yao, K. (1998). Proceso de lexicalización en una lengua no nativa: caso del francés en Costa de Marfil. Thèse doctorale, Valladolid : Université de Valladolid.

Yao, K. (2013). El nouchi, argot, pigdin o criollo. Revista Asia y Africa, 48(2), 537-555.

How to reference this article: Yao, K. (2018). Métaphores et calques dans la création phraséologique du français ivoirien. Íkala, Revista de Lenguaje y Cultura, 23(3), 469-483. DOI : 10.17533/udea.ikala. v23n03a03 